\title{
Preface to the Special Issue on Biophotonics Technologies
}

We are pleased to present the special issue of J-BPE, which focuses on biophotonics technologies for different biomedical applications. The issue contains selected papers presented at Saratov Fall Meeting 2017 (SFM-17) - International Symposium on Optics and Biophotonics - V (September 25-29, 2017, Saratov, Russia) and includes eight representative papers that well characterize the major topics of SFM-17.

Invited paper by Khaksari and Kirkpatrick is devoted to the development of the speckle contrast imaging technique. Basing on the analysis of the first and second order statistics of a single speckle image (single frame) authors give recommendation how to carrying out the speckle measurements.

Invited paper by Carneiro and co-authors discusses the wavelength dependences of the refractive index of healthy and pathological human colorectal tissues. Authors underline that refractive index, which can be measured directly, is a significant optical parameter for the development of optical technologies applicable in clinical practice since it allows one to discriminate healthy and pathological tissues.

The paper by Solovyev et al. deals with investigations of fluorescence of green fluorescent proteins. In this study the authors show for the first time that V127T SAASoti - FP without any point mutations in the chromophore environment can be reversibly photoswitched from the green 'on' to the dark 'off' state that can be useful for development of methods of sub-diffraction microscopy.

Genova et al. present results of investigation and comparison of the macro and micro fluorescence characteristics of ex vivo fresh and fixed tissue samples by fluorescence and synchronous fluorescence spectroscopies, and laser scanning confocal fluorescence microscopy, respectively, in application to differentiation of cancerous and healthy gastrointestinal tissues.

The paper by Bashkatov et al. presents results of ex vivo studies on control of absorption and scattering properties of the human gastric wall mucosa. These studies are able to optimize the laser radiation penetration depth and tissue absorbance, hence, to deliver optimal laser energy for more safe and less invasive therapeutics.

Maryakhina et al. investigate spectral characteristics of benign and malignant breast tissues at different stages of diseases in order to improve the optical techniques of breast cancer diagnostics. As a cheaper and quicker method, it can be used potentially for the breast pathologies screening instead of traditional biopsy and histological examination.

In overall, papers collected in this special issue demonstrate well the exciting potential of optical technologies for biomedical studies and applications aiming medical diagnostics and treatment.

Special Issue Editors:

\section{Alexey N. Bashkatov, Elina A. Genina,}

Optics and Biophotonics Department, Research and Educational Institute of Optics and Biophotonics of Saratov State University (National Research University); Interdisciplinary Laboratory of Biophotonics of Tomsk State University (National Research University), Russia

\section{Valery V. Tuchin,}

Optics and Biophotonics Department, Research and Educational Institute of Optics and Biophotonics of Saratov State University (National Research University); Samara National Research University; Institute of Precision Mechanics and Control RAS, Russia 